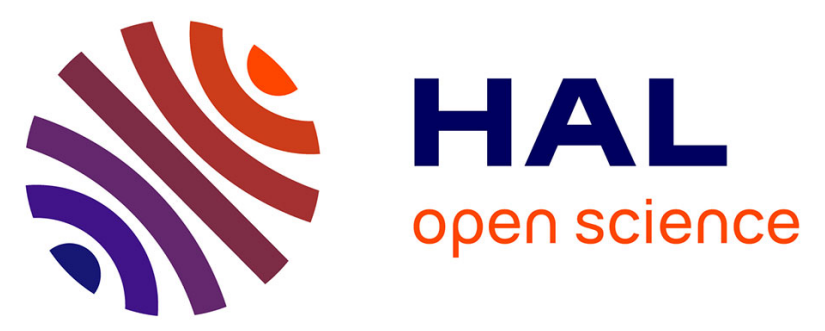

\title{
Analytical Expression of the Magnetic Field Created by Tile Permanent Magnets Tangentially Magnetized and Radial Currents in Massive Disks
}

Romain Ravaud, Guy Lemarquand

\section{- To cite this version:}

Romain Ravaud, Guy Lemarquand. Analytical Expression of the Magnetic Field Created by Tile Permanent Magnets Tangentially Magnetized and Radial Currents in Massive Disks. Progress In Electromagnetics Research B, 2009, 13, pp.309-328. 10.2528/PIERB09012704 • hal-00366542

\section{HAL Id: hal-00366542 https://hal.science/hal-00366542}

Submitted on 9 Mar 2009

HAL is a multi-disciplinary open access archive for the deposit and dissemination of scientific research documents, whether they are published or not. The documents may come from teaching and research institutions in France or abroad, or from public or private research centers.
L'archive ouverte pluridisciplinaire HAL, est destinée au dépôt et à la diffusion de documents scientifiques de niveau recherche, publiés ou non, émanant des établissements d'enseignement et de recherche français ou étrangers, des laboratoires publics ou privés. 


\title{
ANALYTICAL EXPRESSION OF THE MAGNETIC FIELD CREATED BY TILE PERMANENT MAGNETS TANGENTIALLY MAGNETIZED AND RADIAL CURRENTS IN MASSIVE DISKS
}

\author{
R. Ravaud and G. Lemarquand Laboratoire d'Acoustique de \\ l'Universite du Maine, UMR CNRS 6613 \\ Avenue Olivier Messiaen, 72085 Le Mans, France \\ guy.lemarquand@univ-lemans.fr
}

\begin{abstract}
In this paper, we present new expressions for calculating the magnetic field produced by either tile permanent magnets tangentially magnetized or by radial currents in massive disks. These expressions are fully analytical, that is, we do not use any special functions for calculating them. In addition, they are three-dimensional and can be used for calculating the magnetic field for all regular points in space. The expressions commonly used for calculating the magnetic field produced by radial currents in massive disks are often based on elliptic integrals or semi-analytical forms. We propose in this paper an alternative analytical method that can also be used for tile permanent magnets. Indeed, by using the analogy between the coulombian model and the amperian current model, radial currents in massive disks can be represented by using the fictitious magnetic pole densities that are located on two faces of a tile permanent magnet tangentially magnetized. The two representations are equivalent and thus, the shape of magnetic field produced is the same for all points in space, with a smaller value in the case of it is produced by radial currents in massive disks. Such expressions can be used for realizing easily parametric studies.
\end{abstract}

\section{INTRODUCTION}

The calculation of the magnetic field produced by radial currents in massive disks has been studied by S. Babic, C. Akyel, S. J Salon and S. Kincic [1][2]. Indeed, they obtained semi-analytical forms of the magnetic field produced by radial current in massive disks by using the Biot-Savart Law. We propose in this paper to use another approach for calculating the three components of the magnetic field produced by 
radial current in massive disks. Indeed, we use the Coulombian model instead of the Biot-Savart Law. By using the analogy between the coulombian model and the amperian current model, we can show that radial currents in massive disks can be represented as tile permanent magnets whose polarization is perfectly tangential. In this case, the shape of the magnetic field produced by radial current in massive disks is exactly the same as the one produced by a tile permanent magnet tangentially magnetized. However, it is noted that magnetic fields produced by radial currents are always weaker than the ones produced by permanent magnets. Some previous calculations of the magnetic field produced by tile or arc-shaped permanent magnets were determined with the coulombian model [3]-[6]. In addition, other authors have proposed three-dimensional semi-analytical expressions for calculating the magnetic field produced by thick annular conductors [7]-[9]. More generally, such semi-analytical expressions can be employed for optimizing devices using cylindrical permanent magnets [10]-[18]. The expressions obtained are often based on one or two numerical integrations or elliptic integrals [19]-[21]. Consequently, some authors have proposed the use of toroidal functions for the computation of the external magnetic field produced by cylindrical permanent magnets [22]-[24].

The knowledge of the magnetic field produced by arc-shaped permanent magnets is always interesting for the design of actuators or sensors [25]-[36].

This paper is composed in two parts.

The first part deals with the analytical calculation of the magnetic field produced by a tile permanent magnet tangentially magnetized. The three expressions of the magnetic components are determined by using the coulombian model. Studying the magnetic field produced by tile permanent magnets tangentially magnetized can be interesting because such tile permanent magnets can be used for the design of magnetic couplings. We have represented in Fig 1 an example of structure using tile permanent magnets whose polarizations are perfectly tangential but with alternate polarizations. In addition, such tile permanent magnets can be used for the design of electrical machines. Indeed, in a classical electrical machine, the radial field created by the stator constituted by an assembly of tile permanent magnets whose polarizations are often radial can also be optimized by adding tile permanent magnets tangentially magnetized in this structure. This way of assembling tile permanent magnets with several magnetizations allow one to optimize more easily the shape of the radial 


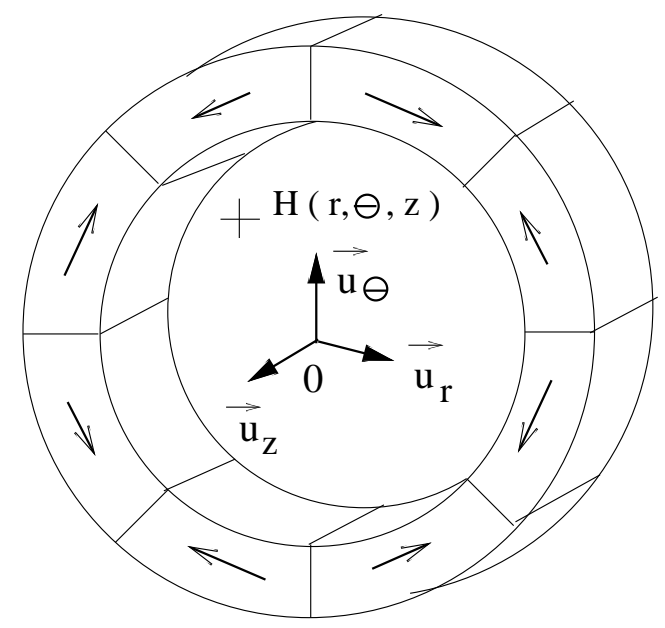

Figure 1. Representation of an assembly of tile permanent magnets tangentially magnetized with alternate polarizations

field produced. Moreover, the magnetic field created by assemblies of tile permanent magnets radially and tangentially magnetized allow one to obtain more intense magnetic fields inside such structures. This is interesting for ironless structures with ferrofluid seals or for realizing magnetic couplings. Furthermore, such tile permanent magnets are used for realizing Halbach structures. These kinds of structures are very interesting because they allow us to create an uniform and intense field inside a ring of tile permanent magnets. Eventually, we think that such tile permanent magnets will be more and more used for realizing unconventional structures of stacked permanent magnets.

In the second part of this paper, we discuss the possibility of using the expressions of the magnetic field components created by a tile permanent magnet tangentially magnetized for the case of massive disks with radial currents. By comparing the coulombian model and the amperian current model, we show that the two calculations are the same. Such an analogy is interesting because the expressions of the magnetic field components produced by a tile permanent magnet tangentially magnetized are fully analytical whereas the ones created by radial currents in massive disks use elliptic integrals and one term must be solved numerically [1]. Consequently, we show that it seems to be possible to obtain a fully analytical expression of the magnetic field produced by radial currents in massive disks. 


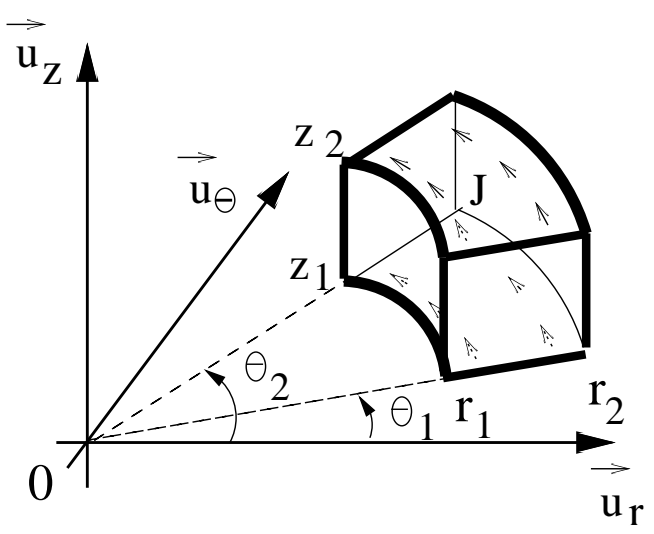

Figure 2. Representation of the geometry considered. The tile inner radius is $r_{1}$, the tile outer radius is $r_{2}$, its angular width is $\theta_{2}-\theta_{1}$. Its magnetic polarization is $\vec{J}$ and its height is $z_{2}-z_{1}$

\section{STUDY OF THE MAGNETIC FIELD PRODUCED BY}

\section{A TILE PERMANENT MAGNET TANGENTIALLY}

\section{MAGNETIZED}

\subsection{Notation and geometry}

The geometry considered and the related parameters are shown in Fig 2. The inner radius of the tile is $r_{1}$, its outer radius is $r_{2}$. Its angular width equals $\theta_{2}-\theta_{1}$ with $\theta_{2}>\theta_{1}$. It height is $z_{2}-z_{1}$ with $z_{2}>z_{1}$. Its magnetic polarization is denoted $\vec{J}$ and verifies for all points in tile tile permanent magnet the following equation:

$$
\vec{J}=J \vec{u}_{\theta}
$$

This implies that the polarization of the tile permanent magnet is perfectly tangential. The magnetic field produced by such a tile permanent magnet can be determined by using the coulombian model. Indeed, according to the Coulombian model, this tile permanent magnet generates a magnetic field that can be deducted from its magnetic potential $\Phi(\vec{r})$ for all points in space. This magnetic potential is given by :

$$
\Phi(\vec{r})=\frac{1}{4 \pi \mu_{0}}\left(\sum_{i} \iint_{\left(S_{i}\right)} \frac{\vec{J} \cdot d \mathbf{S}_{i}}{|\vec{r}-\overrightarrow{\tilde{r}}|}+\iiint_{(V)} \frac{-\vec{\nabla} \cdot \vec{J}}{|\vec{r}-\overrightarrow{\tilde{r}}|} d V\right)
$$




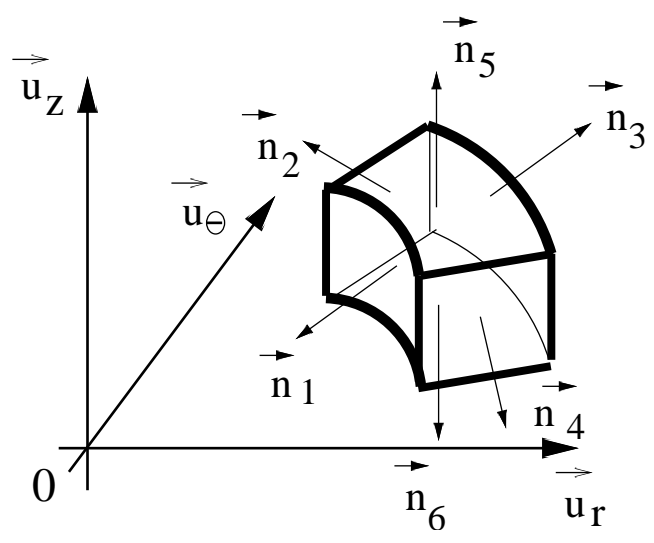

Figure 3. Representation of the tile permanent magnet studied in which the six vector normal units are defined

where $S_{i}$ represents one surface of the tile permanent magnet and $V$ its volume. In addition, $|\vec{r}-\overrightarrow{\widetilde{r}}|$ represents the distance between a point located on the tile permanent magnet and the observation point. Therefore, there is a magnetic pole surface density $\sigma_{s}^{*}$ given by :

$$
\sigma_{s}^{*}=\vec{J} \cdot \vec{n}
$$

where $\vec{n}$ is the unit normal vector. In addition, there is a magnetic pole volume density given by :

$$
\sigma_{v}^{*}=-\vec{\nabla} \cdot \vec{J}
$$

Thus, according to the Coulombian model, a tile permanent magnet can be represented by magnetic pole surface densities that are located on the faces of the magnet and a magnetic pole volume density that is located in the tile permanent magnet. Let us now define the normal vector units of each face of the tile permanent magnet. For this purpose, let consider the figure 3 in which the six vector normal units are defined. We use the notation $\sigma_{s, i}^{*}$ for representing the magnetic pole surface density that appear on the face whose normal unit is $\vec{n}_{i}$. Therefore, we obtain:

$$
\begin{gathered}
\sigma_{s, 1}^{*}=\vec{J} . \vec{n}_{1}=0 \\
\sigma_{s, 2}^{*}=\vec{J} \cdot \vec{n}_{2}=+J \\
\sigma_{s, 3}^{*}=\vec{J} \cdot \vec{n}_{3}=0
\end{gathered}
$$




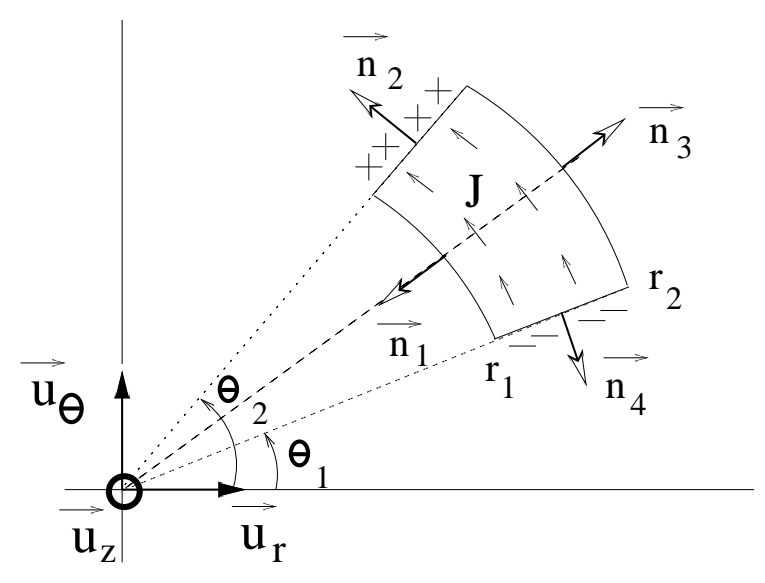

Figure 4. Representation of the geometry considered. The tile inner radius is $r_{1}$, the tile outer radius is $r_{2}$, its angular width is $\theta_{2}-\theta_{1}$. Its magnetic polarization is $\vec{J}$, its height is $z_{2}-z_{1}$

$$
\begin{gathered}
\sigma_{s, 4}^{*}=\vec{J} . \vec{n}_{4}=-J \\
\sigma_{s, 5}^{*}=\vec{J} \cdot \vec{n}_{5}=0 \\
\sigma_{s, 6}^{*}=\vec{J} . \vec{n}_{6}=0
\end{gathered}
$$

The magnetic pole volume density $\sigma_{v}^{*}$ can be determined by using the definition of the divergence in cylindrical coordinates. We deduct that:

$$
\sigma_{v}^{*}=-\vec{\nabla} \cdot \vec{J}=0
$$

Consequently, only two magnetic pole surface densities appear on the tile permanent magnet tangentially magnetized. The first one $-J$ is located on the face whose angular abscissa equals $\theta_{1}$. The second one $+J$ is located on the face whose angular abscissa equals $\theta_{2}$. We show a cross-section of the tile permanent magnet tangentially magnetized with its magnetic pole surface densities in the $(R, \theta)$ plane in Fig 4. 


\subsection{Three-Dimensional analytical expression of the magnetic potential created by the tile permanent magnet tangentially magnetized}

The reciprocal distance, in cylindrical coordinates, between the source point $(\tilde{r}, \tilde{\theta}, \tilde{z})$ and the observation point $(r, \theta, z)$ is expressed as follows:

$$
R(\tilde{r}, \tilde{\theta}, \tilde{z})=\frac{1}{|\vec{r}-\tilde{\vec{r}}|}=\frac{1}{\sqrt{r^{2}+\tilde{r}^{2}-2 r \tilde{r} \cos (\theta-\tilde{\theta})+(z-\tilde{z})^{2}}}
$$

where $\overrightarrow{\tilde{r}}$ represents the position vector of the source point and $\vec{r}$ is the position vector of the observation point. It is emphasized here that this reciprocal distance is not regular for all points in space. There is a discontinuity of this reciprocal distance when the magnetic field is determined on the permanent magnet surfaces. Several approaches can be used for solving this problem. One method has been proposed by [3] with the use of the Lambda's function. Another possibility is to use the Cauchy principal value. By using the Gauss' theorem, the magnetic field value can be easily verified when the observation point is placed on the permanent magnet surfaces. However, it can be noted that the magnetic field is rarely determined on the permanent magnets but rather outside of the tile permanent magnets and also inside the permanent magnet for studying the demagnetizing field. All the analytical expressions presented in this paper can be used for studying the three magnetic field components in the near-field.

Therefore, the magnetic potential $\Phi(r, \theta, z)$ produced by a tile permanent magnet tangentially magnetized is given by:

$$
\begin{aligned}
\Phi(r, \theta, z)= & \frac{J}{4 \pi \mu_{0}} \int_{r_{1}}^{r_{2}} \int_{z_{1}}^{z_{2}} R\left(\tilde{r}, \theta_{2}, \tilde{z}\right) d \tilde{r} d \tilde{z} \\
& -\frac{J}{4 \pi \mu_{0}} \int_{r_{1}}^{r_{2}} \int_{z_{1}}^{z_{2}} R\left(\tilde{r}, \theta_{1}, \tilde{z}\right) d \tilde{r} d \tilde{z}
\end{aligned}
$$

After integrating according to $\tilde{r}$ and $\tilde{z}$, the magnetic potential $\Phi(r, \theta, z)$ can be expressed as follows:

$$
\Phi(r, \theta, z)=\frac{J}{4 \pi \mu_{0}} \sum_{i=1}^{2} \sum_{j=1}^{2} \sum_{k=1}^{2}(-1)^{(i+j+k)} \phi\left(a_{i, j}, b_{i, j}, c_{k}\right)
$$

where

$$
\phi\left(a_{i, j}, b_{i, j}, c_{k}\right)=-z_{k}-\sqrt{b_{i, j}-a_{i, j}^{2}} \arctan \left[\frac{c_{k}}{\sqrt{b_{i, j}-a_{i, j}^{2}}}\right]
$$




$$
\begin{aligned}
& +\sqrt{b_{i, j}-a_{i, j}^{2}} \arctan \left[\frac{a_{i, j} c_{k}}{\sqrt{b_{i, j}-a_{i, j}^{2}} \sqrt{b_{i, j}+c_{k}^{2}}}\right] \\
& -c_{k} \log \left[a_{i, j}+\sqrt{b_{i, j}+c_{k}^{2}}\right] \\
& -a_{i, j} \log \left[c_{k}+\sqrt{b_{i, j}+c_{k}^{2}}\right]
\end{aligned}
$$

and

$$
\begin{gathered}
a_{i, j}=r_{i}-r \cos \left(\theta-\theta_{j}\right) \\
b_{i, j}=r^{2}+r_{i}^{2}-2 r r_{i} \cos \left(\theta-\theta_{j}\right) \\
\xi_{k}=z-z_{k}
\end{gathered}
$$

Eq. (13) is in fact a special case in which the magnetic potential has a fully analytical form. It is emphasized here that this is rarely the case for tile permanent magnets. Though the components of the magnetic field created by tile permanent magnets radially or axially magnetized can often be expressed in terms of elliptic integrals of the first, second and third kind, their magnetic scalar potential is often more difficult to determine in a semi-analytical form. Moreover, it can be easier to determine the magnetic field created by such tile permanent magnets without using the vector or scalar potentials. We present in the next section the expressions of the three components created by the tile permanent magnet tangentially magnetized in fully analytical expressions.

\subsection{Magnetic field produced by the tile permanent magnet tangentially magnetized}

A way of calculating the magnetic field produced by a tile permanent magnet tangentially magnetized is to determine first the magnetic potential $\Phi(r, \theta, z)$ for all points in space. We keep the notation $\xi_{k}$ (Eq. 18) for the equations of the three magnetic components $H_{r}(r, \theta, z)$, $H_{\theta}(r, \theta, z)$ and $H_{z}(r, \theta, z)$.

\subsection{Derivation of the three components}

The magnetic field created by the tile permanent magnet tangentially magnetized can be deducted by calculating the following expression :

$$
\mathbf{H}=-\vec{\nabla} \Phi(r, \theta, z)
$$


and the three components are given respectively by :

$$
\begin{aligned}
& H_{r}(r, \theta, z)=-\vec{\nabla} \Phi(r, \theta, z) \cdot \vec{u}_{r} \\
& H_{\theta}(r, \theta, z)=-\vec{\nabla} \Phi(r, \theta, z) \cdot \vec{u}_{\theta} \\
& H_{z}(r, \theta, z)=-\vec{\nabla} \Phi(r, \theta, z) \cdot \vec{u}_{z}
\end{aligned}
$$

The three expressions obtained are also given in a fully analytical form.

\subsection{Radial component $H_{r}(r, \theta, z)$}

The radial component $H_{r}(r, \theta, z)$ is expressed as follows:

$$
H_{r}(r, \theta, z)=\frac{J}{4 \pi \mu_{0}} \sum_{i=1}^{2} \sum_{j=1}^{2} \sum_{k=1}^{2}(-1)^{(i+j+k)} h_{r}(i, j, k)
$$

where

$$
\begin{aligned}
h_{r}(i, j, k)= & \frac{\left(-e_{i, j}+\cos \left(\theta-\theta_{j}\right)^{2} f_{j}\right)}{\sqrt{f_{j}\left(f_{j}+b_{i, j}\right)}} \tanh ^{-1}\left[\frac{\xi_{k} \sqrt{f_{j}+b_{i, j}}}{\sqrt{f_{j}} \sqrt{b_{i, j}+\xi_{k}^{2}}}\right] \\
& -\cos \left(\theta-\theta_{j}\right)^{2} \log \left[\xi_{k}+\sqrt{b_{i, j}+\xi_{k}^{2}}\right]
\end{aligned}
$$

with

$$
f_{j}=\frac{r}{r_{i}} e_{i, j}=\frac{r}{r_{i}}\left(r r_{i}\left(-1+\cos \left(\theta-\theta_{j}\right)^{2}\right)\right)
$$

We represent in Fig 5 the radial component of the magnetic field produced by a tile permanent magnet tangentially magnetized versus the angle $\theta$ with the following parameters: $r_{1}=0.025 \mathrm{~m}, r_{2}=0.028$ $\mathrm{m}, r=0.024 \mathrm{~m}, \theta_{1}=-\frac{\pi}{16} \mathrm{rad}, \theta_{2}=+\frac{\pi}{16} \mathrm{rad}, z_{1}=0 \mathrm{~m}, z_{2}=0.003 \mathrm{~m}$, $z=0.0015 \mathrm{~m}, J=1 \mathrm{~T}$.

\subsection{Azimuthal component $H_{\theta}(r, \theta, z)$}

The azimuthal component $H_{\theta}(r, \theta, z)$ of the magnetic field produced by a tile permanent maget tangentially magnetized is expressed as follows:

$$
H_{\theta}(r, \theta, z)=\frac{J}{4 \pi \mu_{0}} \sum_{i=1}^{2} \sum_{j=1}^{2} \sum_{k=1}^{2}(-1)^{(i+j+k)} h_{\theta}(i, j, k)
$$




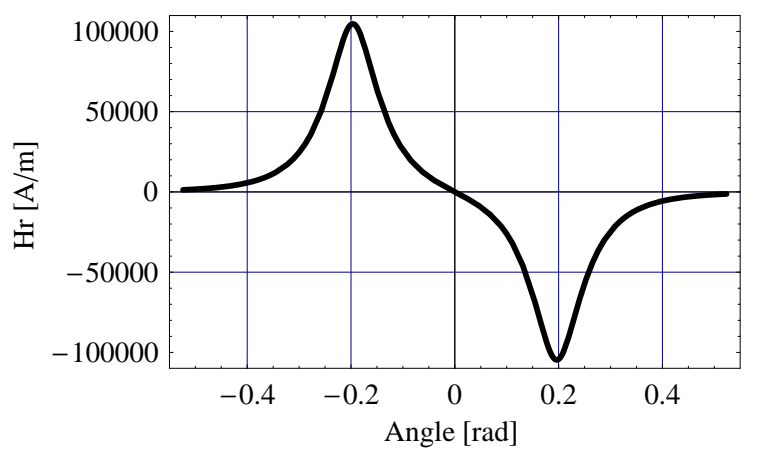

Figure 5. Representation of the radial component $H_{r}$ versus the angle theta $\theta$ with the following parameters: $r_{1}=0.025 \mathrm{~m}, r_{2}=0.028 \mathrm{~m}$, $r=0.024 \mathrm{~m}, \theta_{1}=-\frac{\pi}{16} \mathrm{rad}, \theta_{2}=+\frac{\pi}{16} \mathrm{rad}, z_{1}=0 \mathrm{~m}, z_{2}=0.003 \mathrm{~m}$, $z=0.0015 \mathrm{~m}, J=1 \mathrm{~T}$.

with

$$
\begin{aligned}
h_{\theta}= & -y_{j} \frac{c_{j}+d_{i, j}}{\sqrt{c_{j}\left(b_{i, j}+c_{j}\right)}} \tanh ^{-1}\left[\frac{\xi_{k} \sqrt{b_{i, j}+c_{j}}}{\sqrt{c_{j}\left(b_{i, j}+\xi_{k}^{2}\right)}}\right] \\
& +y_{j} \log \left[\xi_{k}+\sqrt{b_{i, j}+\xi_{k}^{2}}\right]
\end{aligned}
$$

where

$$
\begin{gathered}
y_{j}=\sin \left(\theta-\theta_{j}\right) \\
d_{i, j}=r^{2}-r r_{i} \cos \left(\theta-\theta_{j}\right) \\
c_{j}=r^{2}\left(\left(\cos \left(\theta-\theta_{j}\right)\right)^{2}-1\right)
\end{gathered}
$$

We represent in Fig 6 the azimuthal component of the magnetic field produced by a tile permanent magnet tangentially magnetized versus the angle $\theta$ with the following parameters: $r_{1}=0.025 \mathrm{~m}, r_{2}=0.028 \mathrm{~m}$, $r=0.024 \mathrm{~m}, \theta_{1}=-\frac{\pi}{16} \mathrm{rad}, \theta_{2}=+\frac{\pi}{16} \mathrm{rad}, z_{1}=0 \mathrm{~m}, z_{2}=0.003 \mathrm{~m}$, $z=0.0015 \mathrm{~m}, J=1 \mathrm{~T}$. 


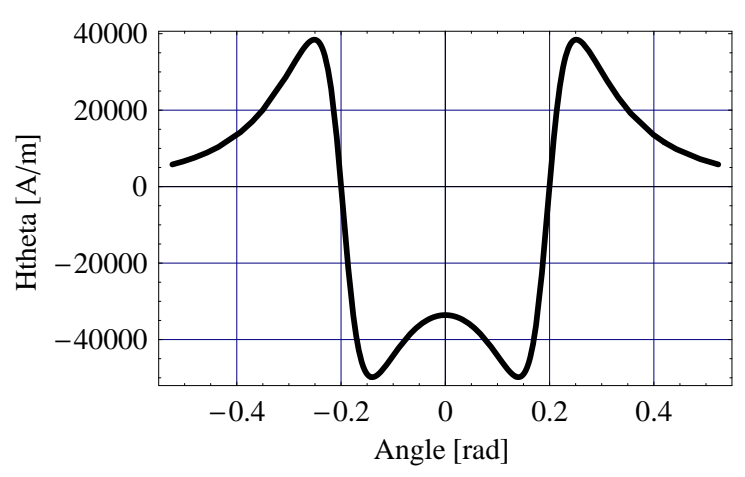

Figure 6. Representation of the azimuthal component $H_{\theta}$ versus the angle theta $\theta$ with the following parameters: $r_{1}=0.025 \mathrm{~m}$, $r_{2}=0.028 \mathrm{~m}, r=0.024 \mathrm{~m}, \theta_{1}=-\frac{\pi}{16} \mathrm{rad}, \theta_{2}=+\frac{\pi}{16} \mathrm{rad}, z_{1}=0 \mathrm{~m}$, $z_{2}=0.003 \mathrm{~m}, z=0.0015 \mathrm{~m}, J=1 \mathrm{~T}$.

\subsection{Axial component $H_{z}(r, \theta, z)$}

The axial component $H_{z}(r, \theta, z)$ of the magnetic field produced by a tile permanent maget tangentially magnetized is expressed as follows:

$$
H_{z}(r, \theta, z)=\frac{J}{4 \pi \mu_{0}} \sum_{i=1}^{2} \sum_{j=1}^{2} \sum_{k=1}^{2}(-1)^{(i+j+k)} h_{z}(i, j, k)
$$

with

$$
h_{z}(i, j, k)=\log \left[r_{i}-r \cos \left(\theta-\theta_{j}\right)+\sqrt{b_{i, j}+\xi_{k}^{2}}\right]
$$

We represent in Fig 7 the axial component of the magnetic field produced by a tile permanent magnet tangentially magnetized versus the angle $\theta$ with the following parameters: $r_{1}=0.025 \mathrm{~m}, r_{2}=0.028 \mathrm{~m}$, $r=0.024 \mathrm{~m}, \theta_{1}=-\frac{\pi}{16} \mathrm{rad}, \theta_{2}=+\frac{\pi}{16} \mathrm{rad}, z_{1}=0 \mathrm{~m}, z_{2}=0.003 \mathrm{~m}$, $z=0.001 \mathrm{~m}, J=1 \mathrm{~T}$.

\subsection{Application of the expressions determined for the study of ironless structures using tile permanent magnets tangentially magnetized}

All the expressions determined previously can be used easily for the study of the magnetic field produced by ironless structures using assemblies of tile permanent magnets tangentially magnetized. Indeed, their computational cost is very low and they are very accurate. We 


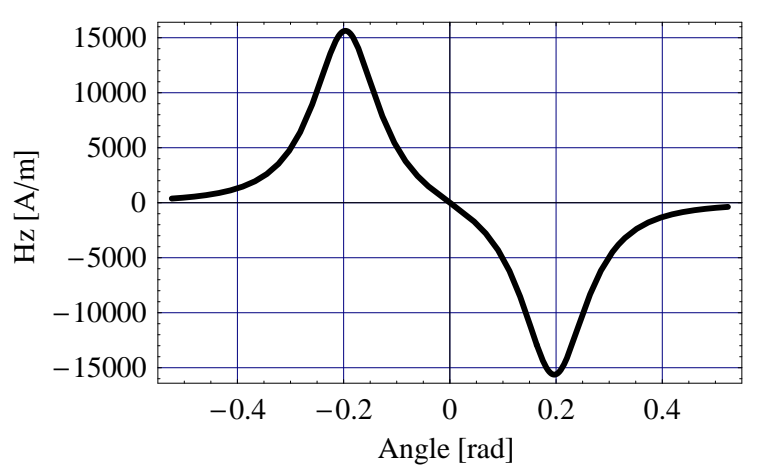

Figure 7. Representation of the axial component $H_{\theta}$ versus the angle theta $\theta$ with the following parameters: $r_{1}=0.025 \mathrm{~m}, r_{2}=0.028 \mathrm{~m}$, $r=0.024 \mathrm{~m}, \theta_{1}=-\frac{\pi}{16} \mathrm{rad}, \theta_{2}=+\frac{\pi}{16} \mathrm{rad}, z_{1}=0 \mathrm{~m}, z_{2}=0.003 \mathrm{~m}$, $z=0.001 \mathrm{~m}, J=1 \mathrm{~T}$.

have represented the three-dimensional representations of the radial and azimuthal components of the magnetic field produced by an assembly of tile permanent magnets tangentially magnetized as shown in Figs 8 and 9 respectively.

\section{MAGNETIC FIELD PRODUCED BY RADIAL CURRENT IN MASSIVE DISKS WITH THE COULOMBIAN MODEL}

We explain in this section how to calculate the magnetic field produced by radial currents in massive disks by using the coulombian model. Several authors have studied the magnetic field produced by such structures [1][9]. In this paper, we study the particular case in which both surface current densities and a volume current density appear in massive disks. Therefore, strictly speaking, our calculation does not treat exactly the same configurations as [1] and [9] but the expressions established in the first part of this paper can also be used for calculating the three components of the magnetic field created by radial currents in massive disks. In fact, there is an analogy between our configuration studied in the first part of this paper and the configurations studied in [1] and [9]. We present in the next section this analogy. 


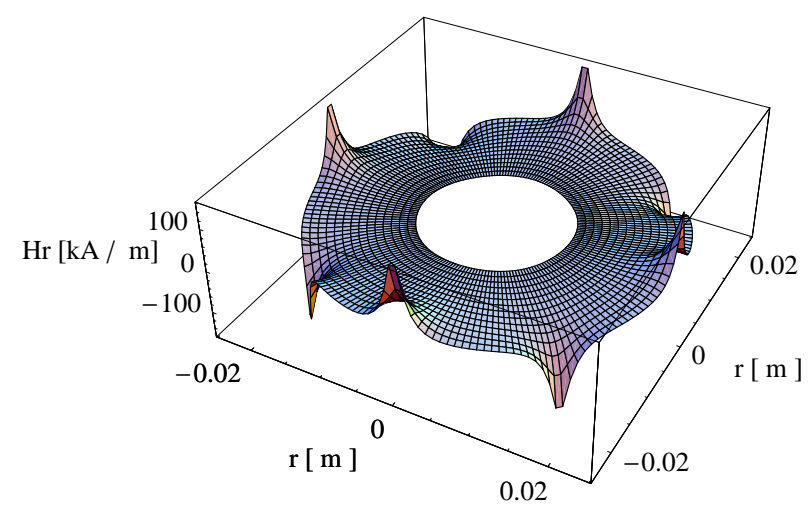

Figure 8. Three-dimensional representation of the radial component $H_{r}$ versus $r$ in polar coordinates with the following parameters: $r_{1}=$ $0.025 \mathrm{~m}, r_{2}=0.028 \mathrm{~m}, z_{1}=0 \mathrm{~m}, z_{2}=0.003 \mathrm{~m}, z=0.001 \mathrm{~m}, J=1 \mathrm{~T}$ and 8 tile permanent magnets are used

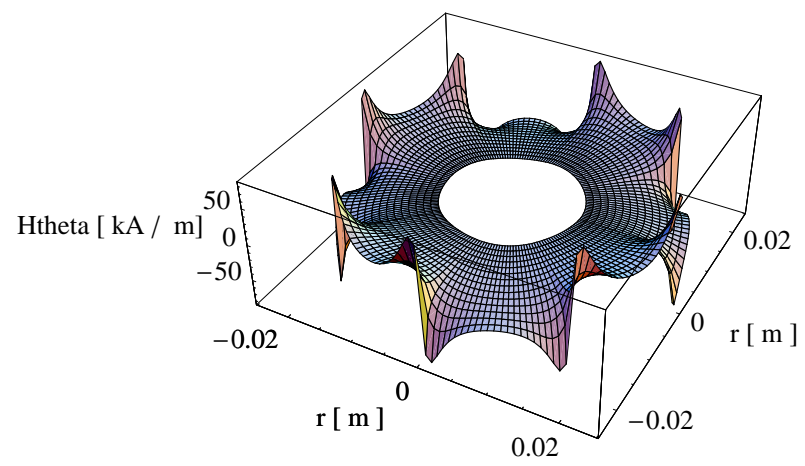

Figure 9. Three-dimensional representation of the azimuthal component $H_{\theta}$ versus $r$ in polar coordinates with the following parameters: $r_{1}=0.025 \mathrm{~m}, r_{2}=0.028 \mathrm{~m}, z_{1}=0 \mathrm{~m}, z_{2}=0.003 \mathrm{~m}$, $z=0.001 \mathrm{~m}, J=1 \mathrm{~T}$ and 8 tile permanent magnets are used 


\subsection{Basic equations}

It is well known that the magnetic field produced by either a permanent magnet or a current distribution can be determined by using either the scalar magnetic potential $\Phi(r, \theta, z)$ or the vector magnetic potential $\vec{A}(r, \theta, z)$. We have shown in the first part that a tile permanent magnet tangentially magnetized generates a scalar magnetic potential for all points in space. Let us now consider the magnetic vector potential created by the tile permanent magnet tangentially magnetized studied in the first part of this paper. This vector magnetic potential $\vec{A}(r, \theta, z)$ that is expressed as follows:

$$
\vec{A}=\frac{\mu_{0}}{4 \pi}\left(\iint_{(S)} \frac{\vec{J}}{\mid \overrightarrow{\mu_{0}} \wedge d \mathbf{S}} \mid \iint_{(V)} \frac{\vec{\nabla} \wedge\left(\frac{\vec{J}}{\mu_{0}}\right) d V}{\left|\vec{r}-\overrightarrow{r^{\prime}}\right|}\right)
$$

Therefore, we can say that there is a surface current density given by :

$$
\overrightarrow{k^{*}}=\frac{\vec{J} \wedge \vec{n}}{\mu_{0}}
$$

In addition, there is a volume current density given by:

$$
\overrightarrow{j^{*}}=\frac{\vec{\nabla} \wedge \vec{J}}{\mu_{0}}
$$

\subsection{Application to a tile permanent magnet tangentially magnetized}

Let us now apply the previous relations the configuration presented in Fig 2. According to the Amperian current model, a tile permanent magnet tangentially magnetized can be represented by using (34) and (35). We find :

$$
\begin{gathered}
k_{S_{1}}=\frac{J}{\mu_{0}} \vec{u}_{\theta} \wedge\left(-\vec{u}_{r}\right)=-\frac{J}{\mu_{0}} \vec{u}_{z} \\
k_{S_{2}}=\frac{J}{\mu_{0}} \vec{u}_{\theta} \wedge \vec{u}_{\theta}=0 \\
k_{S_{3}}=\frac{J}{\mu_{0}} \vec{u}_{\theta} \wedge\left(\vec{u}_{r}\right)=+\frac{J}{\mu_{0}} \vec{u}_{z} \\
k_{S_{4}}=\frac{J}{\mu_{0}} \vec{u}_{\theta} \wedge\left(-\vec{u}_{\theta}\right)=0
\end{gathered}
$$




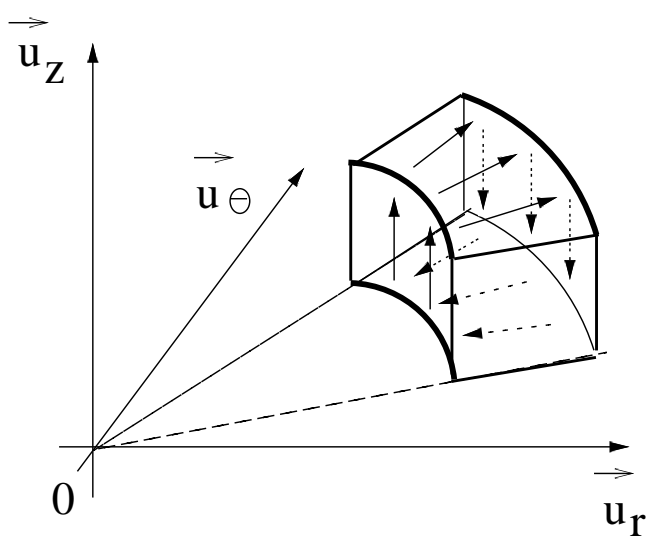

Figure 10. Representation of the surface current densities that appear on a tile permanent magne tangentially magnetized with the amperian current model

$$
\begin{gathered}
k_{S_{5}}=\frac{J}{\mu_{0}} \vec{u}_{\theta} \wedge\left(\vec{u}_{z}\right)=+\frac{J}{\mu_{0}} \vec{u}_{r} \\
k_{S_{6}}=\frac{J}{\mu_{0}} \vec{u}_{\theta} \wedge\left(-\vec{u}_{z}\right)=-\frac{J}{\mu_{0}} \vec{u}_{r}
\end{gathered}
$$

In addition, the volume current density is expressed as follows:

$$
\overrightarrow{j^{*}}=\frac{\vec{\nabla}}{\mu_{0}} \wedge J \vec{u}_{\theta}=\frac{1}{\mu_{0}} \frac{1}{r}\left(\frac{\partial(r J)}{\partial r}\right) \vec{u}_{z}=\frac{J}{r} \vec{u}_{z}
$$

We represent in Fig 10 the surface current densities that are located on four faces of the tile permanent magnet and in Fig 11 the surface current density that is located in the tile permanent magnet. An interesting point to say is that current on the face $S_{1}$ and the current on the face $S_{3}$ are not the same because $S_{3}>S_{1}$. This is why we can say that a volume current density appears inside the tile permanent magnet. This allows us to obtain a charge equilibrium of the magnet. Therefore, the three magnetic components can also be determined by using the following relation:

$$
\begin{aligned}
& H_{r}(r, \theta, z)=\frac{\vec{\nabla}}{\mu_{0}} \wedge \vec{A}(r, \theta, z) \cdot \vec{u}_{r} \\
& H_{\theta}(r, \theta, z)=\frac{\vec{\nabla}}{\mu_{0}} \wedge \vec{A}(r, \theta, z) \cdot \vec{u}_{\theta}
\end{aligned}
$$




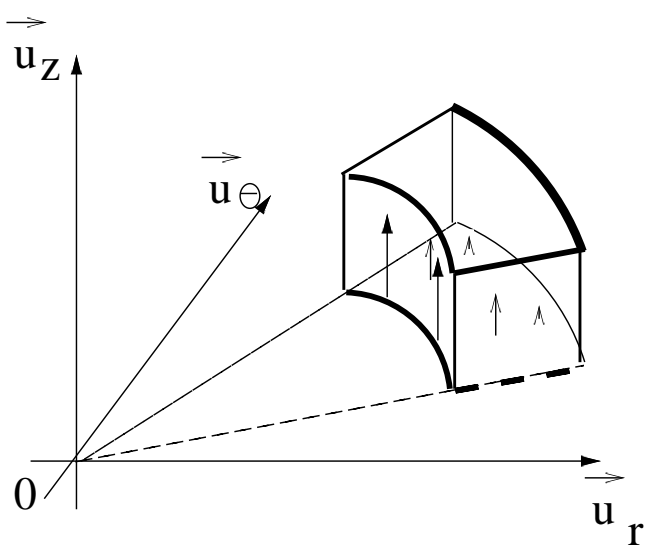

Figure 11. Representation of the volume current density that appears inside a tile permanent magne tangentially magnetized with the amperian current model

$$
H_{z}(r, \theta, z)=\frac{\vec{\nabla}}{\mu_{0}} \wedge \vec{A}(r, \theta, z) \cdot \vec{u}_{z}
$$

In short, by assuming that there are radial currents in massive disks that are characterized by both surface current densities $k^{*}$ and volume current densities $j^{*}$, we can use directly the coulombian model by using the transformations $j^{*}=\frac{\vec{\nabla} \wedge \vec{J}}{\mu_{0}}$ and $k^{*}=\frac{\vec{J} \wedge \vec{n}}{\mu_{0}}$. These two transformations allow us to define the equivalent value of $|\vec{J}|$ that correspond to a tile permanent magnet tangentially magnetized. The interest of such an approach lies in the fact that the expressions of the three magnetic components created by a tile permanent magnet tangentially magnetized are fully analytical. Consequently, we can also find in a fully analytical form the three magnetic components created by radial currents in massive disks.

\subsection{Illustration: comparison with the Biot-Savart Law}

The relations determined in the previous section can be compared with the well-known Biot-Savart Law. For this purpose, let us consider the previous configuration shown in Figs. 10 and 11 in which both a volume current density $\vec{j}^{*}$ and four surface current densities $k_{S_{1}}, k_{S_{3}}, k_{S_{5}}$ and $k_{S_{6}}$ are considered. It is emphasized here that this configuration differs from the one studied in [1] and [9]. The magnetic field produced by 
such a current distribution is given by:

$$
\begin{aligned}
& \vec{H}(\vec{r})=\frac{1}{4 \pi} \int_{r_{1}}^{r_{2}} \int_{\theta_{1}}^{\theta_{2}} \int_{z_{1}}^{z_{2}} \frac{\overrightarrow{j^{*}} d v \wedge\left(\vec{r}-\overrightarrow{r^{\prime}}\right)}{\left|\vec{r}-\overrightarrow{r^{\prime}}\right|^{3}} \\
& +\frac{1}{4 \pi} \int_{\theta_{1}}^{\theta_{2}} \int_{z_{1}}^{z_{2}} \frac{\vec{k}_{S_{1}} d S_{1} \wedge\left(\vec{r}-\overrightarrow{r^{\prime}}\right)}{\left|\vec{r}-\overrightarrow{r^{\prime}}\right|^{3}} \\
& +\frac{1}{4 \pi} \int_{\theta_{1}}^{\theta_{2}} \int_{z_{1}}^{z_{2}} \frac{\vec{k}_{S_{3}} d S_{3} \wedge\left(\vec{r}-\overrightarrow{r^{\prime}}\right)}{\left|\vec{r}-\overrightarrow{r^{\prime}}\right|^{3}} \\
& +\frac{1}{4 \pi} \int_{r_{1}}^{r_{2}} \int_{\theta_{1}}^{\theta_{2}} \frac{\vec{k}_{S_{5}} d S_{5} \wedge\left(\vec{r}-\overrightarrow{r^{\prime}}\right)}{\left|\vec{r}-\overrightarrow{r^{\prime}}\right|^{3}} \\
& +\frac{1}{4 \pi} \int_{r_{1}}^{r_{2}} \int_{\theta_{1}}^{\theta_{2}} \frac{\vec{k}_{S_{6}} d S_{6} \wedge\left(\vec{r}-\overrightarrow{r^{\prime}}\right)}{\left|\vec{r}-\overrightarrow{r^{\prime}}\right|^{3}}
\end{aligned}
$$

For our numerical simulation, we take a surface current density $\left|\vec{k}_{S_{i}}\right|$ that equals $10^{4} \mathrm{~A} / \mathrm{m}^{2}$ and $i$ is the surface $i$ of the tile, $r_{1}=0.025 \mathrm{~m}$, $r_{2}=0.028 \mathrm{~m}, z_{1}=0 \mathrm{~m}, z_{2}=0.003 \mathrm{~m}, \theta_{1}=0 \mathrm{rad}, \theta_{2}=\frac{\pi}{8} \mathrm{rad}$, and $\left|\overrightarrow{j^{*}}\right|$ that equals $\frac{10^{4}}{r} \mathrm{~A} / \mathrm{m}^{3}, \theta=\frac{\pi}{7} \mathrm{rad}, z=0 \mathrm{~m}$. We use the magnetic induction field $\vec{B}$ for the simulations and its calculation has been performed by numerical means. We represent in Fig. 12 the radial component of the magnetic induction field versus the radial observation point with the previous parameter values. Figure 12 confirms the validity of the coulombian model for calculating the magnetic field produced by radial currents in massive disks.

\section{CONCLUSION}

We have presented new expressions for calculating the magnetic field produced by either a tile permanent magnet tangentially magnetized, or by radial currents in massive disks. All the expressions determined in this paper are presented in a fully analytical form, that is, without any special functions as elliptic integrals. Consequently, such expressions have a very low computational cost and are accurate because all the expressions have been determined without any simplifying assumptions. The analogy between the coulombian model 


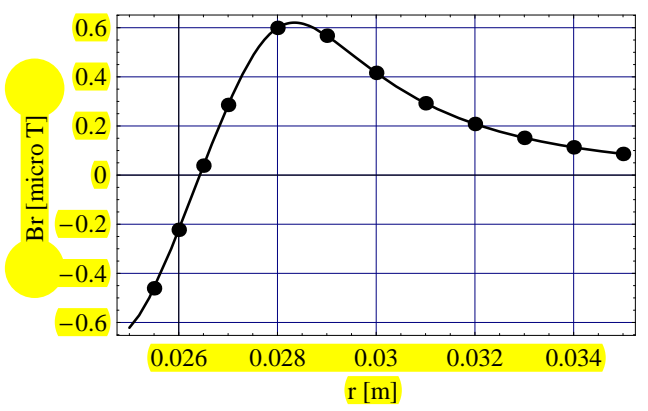

Figure 12. Representation of the radial component of the magnetic induction field $\vec{B}$ determined with the coulombian model (thick line) and with the Biot-Savart Law (points). We take $\left|\vec{k}_{S_{i}}\right|=10^{4} \mathrm{~A} / \mathrm{m}^{2}$ where $i$ is the surface $i$ of the tile, $r_{1}=0.025 \mathrm{~m}, r_{2}=0.028 \mathrm{~m}$, $z_{1}=0 \mathrm{~m}, z_{2}=0.003 \mathrm{~m}, \theta_{1}=0 \mathrm{rad}, \theta_{2}=\frac{\pi}{8} \mathrm{rad},\left|\overrightarrow{j^{*}}\right|=\frac{10^{4}}{r} \mathrm{~A} / \mathrm{m}^{3}$, $\theta=\frac{\pi}{7} \mathrm{rad}, z=0 \mathrm{~m}$.

and the amperian current model allows us to calculate with the same expressions the magnetic field produced by tile permanent magnets whose polarization is tangential or by radial currents in massive disks.

\section{REFERENCES}

1. S. Babic, C. Akyel, S. Salon, and S. Kincic, "New expressions for calculating the magnetic field created by radial current in massive disks," IEEE Trans. Magn., vol. 38, no. 2, pp. 497-500, 2002.

2. S. Babic and M. M. Gavrilovic, "New expression for calculating magnetic fields due to current-carrying solid conductors," IEEE Trans. Magn., vol. 33, no. 5, pp. 4134-4136, 1997.

3. S. Babic and C. Akyel, "Improvement in the analytical calculation of the magnetic field produced by permanent magnet rings," Progress in Electromagnetics Research C, vol. 5, pp. 71-82, 2008.

4. R. Ravaud, G. Lemarquand, V. Lemarquand, and C. Depollier, "Analytical calculation of the magnetic field created by permanent-magnet rings," IEEE Trans. Magn., vol. 44, no. 8, pp. 1982-1989, 2008.

5. R. Ravaud, G. Lemarquand, V. Lemarquand, and C. Depollier, "The three exact components of the magnetic field created 
by a radially magnetized tile permanent magnet.," Progress in Electromagnetics Research, PIER 88, pp. 307-319, 2008.

6. R. Ravaud, G. Lemarquand, V. Lemarquand, and C. Depollier, "Discussion about the analytical calculation of the magnetic field created by permanent magnets.," Progress in Electromagnetics Research B, vol. 11, pp. 281-297, 2009.

7. B. Azzerboni and E. Cardelli, "Magnetic field evaluation for disk conductors," IEEE Trans. Magn., vol. 29, no. 6, pp. 2419-2421, 1993.

8. B. Azzerboni, E. Cardelli, M. Raugi, A. Tellini, and G. Tina, "Magnetic field evaluation for thick annular conductors," IEEE Trans. Magn., vol. 29, no. 3, pp. 2090-2094, 1993.

9. B. Azzerboni and G. Saraceno, "Three-dimensional calculation of the magnetic field created by current-carrying massive disks," IEEE Trans. Magn., vol. 34, no. 5, pp. 2601-2604, 1998.

10. E. P. Furlani, Permanent Magnet and Electromechanical Devices: Materials, Analysis and Applications. Academic Press, 2001.

11. E. P. Furlani, S. Reznik, and A. Kroll, "A three-dimensonal field solution for radially polarized cylinders," IEEE Trans. Magn., vol. 31, no. 1, pp. 844-851, 1995.

12. E. P. Furlani and M. Knewston, "A three-dimensional field solution for permanent-magnet axial-field motors," IEEE Trans. Magn., vol. 33, no. 3, pp. 2322-2325, 1997.

13. E. P. Furlani, "A two-dimensional analysis for the coupling of magnetic gears," IEEE Trans. Magn., vol. 33, no. 3, pp. 23172321, 1997.

14. E. P. Furlani, "Field analysis and optimization of ndfeb axial field permanent magnet motors," IEEE Trans. Magn., vol. 33, no. 5, pp. 3883-3885, 1997.

15. D. Mayergoyz and E. P. Furlani, "The computation of magnetic fields of permanent magnet cylinders used in the electrophotographic process.," J. Appl. Phys., vol. 73, no. 10, pp. 5440-5442, 1993.

16. P. Elies and G. Lemarquand, "Analytical optimization of the torque of a permanent-magnet coaxial synchronous coupling," IEEE Trans. Magn., vol. 34, no. 4, pp. 2267-2273, 1998.

17. V. Lemarquand, J. F. Charpentier, and G. Lemarquand, "Nonsinusoidal torque of permanent-magnet couplings," IEEE Trans. Magn., vol. 35, no. 5, pp. 4200-4205, 1999.

18. M. Lang, "Fast calculation method for the forces and stiffnesses of permanent-magnet bearings," 8th International Symposium on 
Magnetic Bearing, pp. 533-537, 2002.

19. S. Babic and C. Akyel, "An improvement in the calculation of the magetic field for an arbitrary geometry coil with rectangular cross section," International Journal of Numerical Modelling: Electronic Networks, Devices and Fields, vol. 18, pp. 493-504, November 2005.

20. S. Babic, C. Akyel, and S. Salon, "New procedures for calculating the mutual inductance of the system: filamentary circular coilmassive circular solenoid," IEEE Trans. Magn., vol. 39, no. 3, pp. 1131-1134, 2003.

21. S. Babic, C. Akyel, and M. M. Gavrilovic, "Calculation improvement of 3d linear magnetostatic field based on fictitious magnetic surface charge," IEEE Trans. Magn., vol. 36, no. 5, pp. $3125-3127,2000$.

22. J. P. Selvaggi, S. Salon, O. M. Kwon, M. V. K. Chari, and M. DeBortoli, "Computation of the external magnetic field, nearfield or far-field from a circular cylindrical magnetic source using toroidal functions," IEEE Trans. Magn., vol. 43, no. 4, pp. 11531156, 2007.

23. J. P. Selvaggi, S. Salon, O. M. Kwon, and M. V. K. Chari, "Computation of the three-dimensional magnetic field from solid permanent-magnet bipolar cylinders by employing toroidal harmonics," IEEE Trans. Magn., vol. 43, no. 10, pp. 3833-3839, 2007.

24. J. P. Selvaggi, S. Salon, O. M. Kwon, and M. V. K. Chari, "Calculating the external magnetic field from permanent magnets in permanent-magnet motors - an alternative method," IEEE Trans. Magn., vol. 40, no. 5, pp. 3278-3285, 2004.

25. R. Ravaud, G. Lemarquand, V. Lemarquand, and C. Depollier, "Ironless loudspeakers with ferrofluid seals," Archives of Acoustics, vol. 33, no. 4, pp. 3-10, 2008.

26. J. Wang, G. W. Jewell, and D. Howe, "Design optimisation and comparison of permanent magnet machines topologies," vol. 148, pp. 456-464, IEE.Proc.Elect.Power Appl., 2001.

27. J. P. Yonnet, "Permanent magnet bearings and couplings," IEEE Trans. Magn., vol. 17, no. 1, pp. 1169-1173, 1981.

28. Z. Zhu, G. W. Jewell, and D. Howe, "Design considerations for permanent magnet polarised electromagnetically actuated brakes," IEEE Trans. Magn., vol. 31, no. 6, pp. 3743-3745, 1995.

29. M. Abele, J. Jensen, and H. Rusinek, "Generation of uniform high fields with magnetized wedges," IEEE Trans. Magn., vol. 33, 
no. 5, pp. 3874-3876, 1997.

30. W. Baran and M. Knorr, "Synchronous couplings with sm co5 magnets," pp. 140-151, 2nd Int. Workshop on Rare-Earth Cobalt Permanent Magnets and Their Applications, Dayton, Ohio, USA, 1976.

31. M. Remy, G. Lemarquand, B. Castagnede, and G. Guyader, "Ironless and leakage free voice-coil motor made of bonded magnets," IEEE Trans. Magn., vol. 44, no. 11, 2008.

32. M. Berkouk, V. Lemarquand, and G. Lemarquand, "Analytical calculation of ironless loudspeaker motors," IEEE Trans. Magn., vol. 37, no. 2, pp. 1011-1014, 2001.

33. C. Blache and G. Lemarquand, "High magnetic field gradients in flux confining permanent magnet structures," Journal of Magnetism and Magnetic Materials, vol. 104, pp. 1111-1112, 1992.

34. C. Blache and G. Lemarquand, "New structures for linear displacement sensor with hight magnetic field gradient," IEEE Trans. Magn., vol. 28, no. 5, pp. 2196-2198, 1992.

35. J. F. Charpentier and G. Lemarquand, "Optimization of unconventional p.m. couplings," IEEE Trans. Magn., vol. 38, no. 2, pp. 1093-1096, 2002.

36. G. Lemarquand, "Ironless loudspeakers," IEEE Trans. Magn., vol. 43, no. 8, pp. 3371-3374, 2007. 